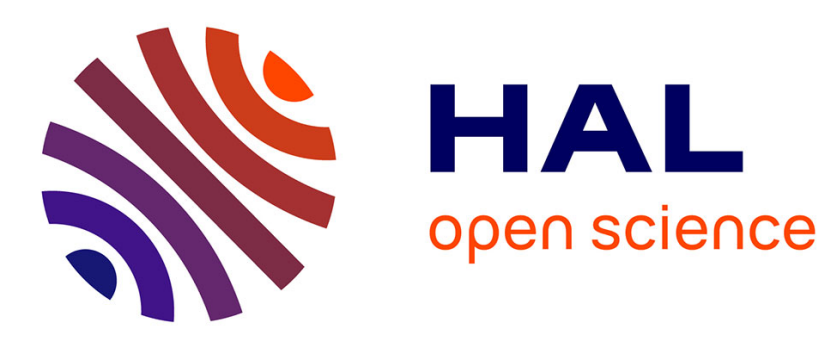

\title{
Networked control under time-synchronization errors
}

Alexandre Seuret, Karl Henrik Johansson

\section{To cite this version:}

Alexandre Seuret, Karl Henrik Johansson. Networked control under time-synchronization errors. Sipahi, R.; Vyhlídal, T.; Niculescu, S.-I.; Pepe, P.; Rifat Sipahi; Tomáš Vyhlídal; Silviu-Iulian Niculescu; Pepe Pierdomenico. Time Delay Systems: Methods, Applications and New Trends, 423, Springer, pp.369-383, 2012, Lecture Notes in Control and Information Sciences, 978-3-642-25220-4. hal-00677291

\section{HAL Id: hal-00677291 \\ https://hal.science/hal-00677291}

Submitted on 7 Mar 2012

HAL is a multi-disciplinary open access archive for the deposit and dissemination of scientific research documents, whether they are published or not. The documents may come from teaching and research institutions in France or abroad, or from public or private research centers.
L'archive ouverte pluridisciplinaire HAL, est destinée au dépôt et à la diffusion de documents scientifiques de niveau recherche, publiés ou non, émanant des établissements d'enseignement et de recherche français ou étrangers, des laboratoires publics ou privés. 


\title{
Networked control under time-synchronization errors
}

\author{
Alexandre Seuret* and Karl H. Johansson \\ NeCS Team, Automatic Control Department \\ GIPSA-Lab, UMR CNRS 5216, \\ Grenoble. \\ ACCESS Linnaeus Center, Royal Institute of Technology, \\ Stockholm, Sweden
}

\begin{abstract}
A robust controller is derived for networked control systems with uncertain plant dynamics. The link between the nodes is disturbed by time-varying communication delays, samplings and timesynchronization. A stability criterion for a robust control is presented in terms of LMIs based on Lyapunov-Krasovskii techniques. A second-order system example is considered and the relation between the admissive bounds of the synchronization error and the size of the uncertainties is computed.
\end{abstract}

Keywords: Networked control systems, Time-delay, clocks synchronization errors, Lyapunov-Krasovskii functionals

\section{Introduction}

Internet technology appears as a natural and cheap way to ensure the communication link in remotely controlled systems $[1,8,16]$. Today, the available Quality of Service is often good enough for that kind of applications. However, such a communication link constitutes an additional dynamical system, which great influence on stability was already mentioned in the 60's [4]. Indeed, several dynamics and perturbations (communication delay, real-time sampling, packet dropout and synchronization errors) are unavoidably introduced and have to be taken into account during the design of the control/observation loop.

In the literature, many authors assume that the nodes of the NCS are synchronized [8]. However the synchronization is an fundamental issue of NCS since ensuring several nodes are synchronized is not easy and some error in it may reduce the performances of the controller [5]. The article focusses on the lake of time-synchronization and provides a robust controller for continuous networked control systems with synchronization error and to parameter uncertainties. A time-delay representation which takes into account the transmission delays, the sampling and the synchronization errors.

\footnotetext{
* This work was supported by the European project FeedNetBack (http://www.feednetback.eu/)
} 
Several works on networked controlled systems introduced the question of transmission delays [2]. It is well known that delays generally lead to unstable behavior [10][11]. Moreover in networked control situations, the delays are basically variable (jitter phenomenon) and unpredictable. This is a source of problem when the classical predictor-based controllers are intended to be applied. These techniques generally need the constant delay, i.e. $h_{i}(t)=h_{i}$. In the case of variable delays, some researches have used independent-of-delay conditions. Because such i.o.d. conditions may be conservative in general, particular cases such as constant or symmetric delays were considered [3]. These assumptions refers to the case where the transmission delays are equal, i.e. $h_{1}(t)=h_{2}(t)=R(t) / 2$, where $R(t)$ denotes the round trip time (RTT). Another interesting approach was recently given in [14], which generalized the predictor techniques to the case of variable delays.

Considering unknown time-varying delays and samplings, some stability and stabilization results, [15] have been provided known introducing bounds of the delays and of the sampling interval $\left(h_{m}, h_{M}\right.$ and $T$ such that $0 \leq h_{m} \leq h(t) \leq$ $h_{M}$ and such that the difference between two successive sampling instants is less than $T$ ), which is not that restrictive. In this paper, the same assumptions are done to ensure the stability of the NCS using a observer-based controller which extends the controller from [9] to the case of time varying delays, synchronization errors and parameter uncertainties.

The present article is organized as follows. Section II concerns the problem formulation providing a presentation of the plant and of the communication. Section III exposes the control strategy. Section IV deals with the stability of the controller. An example is provided in Section V.

\section{Preliminaries}

The network control problem is described in Fig.1. The plant and the controller are connected through a network which induces additional dynamics. It is assumed that the time synchronization of the process and controller clocks is not achieved. Then the time $t_{p}$ given by the plant's clock and the time $t_{c}$ delivered by the controller's clock do not have the same sense. The reference time is given by the plant clock. It means that $t_{c}=t_{p}+\epsilon(t)$ where $\epsilon$ corresponds to a timevarying error of synchronization. The features of the plant and the assumptions on the network are described in the following.

\subsection{Definition of the plant}

Consider the uncertain systems:

$$
\begin{aligned}
& \dot{x}(t)=\left(A+\Delta_{\gamma} A\right) x(t)+\left(B+\Delta_{\gamma} B\right) u(t), \\
& y(t)=\left(C+\Delta_{\gamma} C\right) x(t) .
\end{aligned}
$$

where $x \in R^{n}, u \in R^{m}$ and $y \in R^{p}$ are, respectively, the state, input and output vectors. The constant and known matrices $A, B$ and $C$ correspond to the 


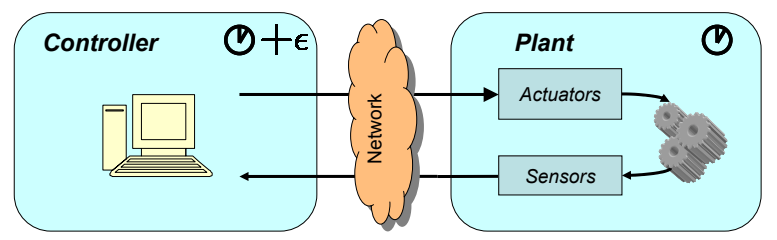

Fig. 1: Plant controller through a network

nominal behavior of the plant. The (time-varying) uncertainties are given in a polytopic representation:

$$
\Delta_{\gamma} A=\gamma \sum_{i=1}^{N} \lambda_{i}(t) A_{i}, \Delta_{\gamma} B=\gamma \sum_{i=1}^{N} \lambda_{i}(t) B_{i} \Delta_{\gamma} C=\gamma \sum_{i=1}^{N} \lambda_{i}(t) C_{i}
$$

where $N$ corresponds to the numbers of vertices. The matrices $A_{i}, B_{i}$ and $C_{i}$ are constant and known. The scalar $\gamma \in R$ characterizes the size of the uncertainties. Note that when $\gamma=0$, no parameter uncertainty is disturbing the system. However the greater the $\gamma$, the greater the disturbances. The functions $\lambda_{i}($.$) are weighted scalar functions which follow a convexity property, ie. for all$ $i=1, . ., N$ and for all $t \geq 0: \lambda_{i}(t) \geq 0$ and $\sum_{i=1}^{N} \lambda_{i}(t)=1$. It is also assumed that the computation power is low on the plant and its functions are limited to receive control packets, to apply control and to send output measurement data. The computation thus is removed in a centralized controller.

\subsection{Synchronization and delays models}

In addition to parameter uncertainties, the stability of the closed-loop system must be ensured whatever the delays, the possible aperiodicity of the real-time sampling processes and synchronization error. Concerning the transmission delays, the delays are assumed to be non-symmetric but have known minimal and maximal bounds $h_{m}$ and $h_{M}$, so that:

$$
\text { A1 (maximal allowed delay) : } h_{m} \leq h_{i}(t) \leq h_{M} .
$$

Since we aim at limiting the value of $h_{m}$, the use of the User Datagram Protocol (UDP) is preferred to Transmission Control Protocol (TCP), the reliability mechanisms of which may needlessly slow down the feedback loop. Another feature of UDP is that the packets do not always arrive in their chronological emission order. The reception function will be added a re-ordering mechanism thanks to some "time-stamps" added in packets. This can be expressed as:

$$
\text { A2 (packet reordering) : } \quad \dot{h}_{i}(t)<1 .
$$

Another disturbance implied by the network comes from the samplers and zero-holders. Following the lines of [6], we consider they produce an additional variable delay $t-t_{k}$, where $t_{k}$ is the $k^{t h}$ sampling instant. Moreover, because of 


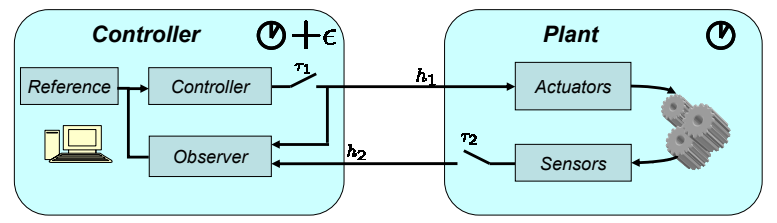

Fig. 2: Architecture of the networked controller

the operating system, the sampling is generally not periodic. So, we only assume there exists a known maximum sampling interval $T$ so that:

$$
\text { A3 (max. sampling interval) : } 0 \leq t_{k+1}-t_{k} \leq T \text {. }
$$

Assume the function $\epsilon$ is time-varying and there exists a known constant $\bar{\epsilon}$ such that:

$$
\mathbf{A} 4:|\epsilon(t)| \leq \bar{\epsilon}
$$

\section{Observer-based networked control}

The system architecture is exposed in Fig. 2. The controller has to estimate present state of plant, using output measurements, and to compute the control value which will be sent to the plant.

D1 The control law: The controller computes a control law which considers some set-values to be reached. The static state feedback control $u(t)=K \hat{x}(t)$ is defined considering the state estimate $\hat{x}$ given by the observer. The difficulty is to determine a gain $K$ guaranteeing stability despite the delay $\delta_{1}(t)$.

D2 Transmission of the control $u$ : The $k^{t h}$ packets sent by the controller to the process includes the designed control $u\left(t_{1, k}\right)$ and a sampling time $t_{1, k}$ when it was produced. The plant receives this information at time $t_{1, k}^{r}$. This time does not have the same meaning for both parts. The term $t_{1, k}^{r}-t_{1, k}$, corresponding to the transmission delay, corrupted by $\epsilon$, is estimated by the plant once the packet has reached it.

D3 Receipt and processing of the control data: The control, sent at time $t_{1, k}$, is received by the process at time $t_{1, k}^{r} \geq t_{1, k}+h_{m}$. There is no raison that the controller also knows the time $t_{1, k}^{r}$ when the control $u\left(t_{1, k}\right)$ will be injected into the plant input. Finally, there exists $k$ such that $h_{m} \leq t_{1, k} \leq$ $h_{M}+T$ and the process is governed by:

$$
\dot{x}(t)=\left(A+\Delta_{\gamma} A\right) x(t)+\left(B+\Delta_{\gamma} B\right) u\left(t_{1, k}\right)
$$

D4 Transmission of the output information: The process have access to its output $y$ only in discrete-time. A packet contains the output $y\left(t_{2, k^{\prime}}\right)$ and the sampling time $t_{2, k^{\prime}}$. The controller receives the output packet at time $t_{2, k^{\prime}}^{r}$. 
D5 Observation of the process: For a given $\hat{k}$ and any $t \in\left[t_{1, \hat{k}}+\left(h_{M}-\right.\right.$ $\left.h_{m}\right) / 2, t_{1, \hat{k}+1}+\left(h_{M}-h_{m}\right) / 2\left[\right.$, there exists a $k^{\prime}$ such that:

$$
\begin{aligned}
& \dot{\hat{x}}(t)=A \hat{x}(t)+B u\left(t_{1, \hat{k}}+\epsilon\right)-L\left(y\left(t_{2, k^{\prime}}\right)-\hat{y}\left(t_{2, k^{\prime}}-\epsilon\right)\right), \\
& \hat{y}(t)=C \hat{x}(t) .
\end{aligned}
$$

The design of an observer gain $L$ ensuring stability is not straightforward.

Note that the observation is based on the nominal values of the system definition. No assumption is introduced to estimate the uncertainties and the $\lambda_{i}$ functions. The time stamp $t_{1, \hat{k}}$ corresponds to the time where the control input is assumed to be implemented into the plant input. The index $k^{\prime}$ corresponds to the most recent output information the controller has received. The time $t_{1, k}^{r}$ and the control $u\left(t_{1, k}\right)$ (see D2) are not known from the observer.

An improve with respect to [13] is that no buffers are required in the controller. This allows considering the input packets as soon as they arrive.

\section{Stabilization under synchronization error}

This section focusses on developing asymptotic stability of the networked control architecture detailed in Fig. 2.

\subsection{Closed-loop system}

The input delay approach to sampled-data signals allows a homogenized definition of the delays $\delta_{1}(t) \triangleq t-t_{1, k}$ where $k$ corresponds to the real sampling implemented in the plant, $\hat{\delta}_{1}(t) \triangleq t-t_{1, \hat{k}}$ and $\delta_{2}(t) \triangleq t-t_{2, k^{\prime}}$ to be considered. The observer dynamics are then driven by:

$$
\begin{aligned}
& \dot{\hat{x}}(t)=A \hat{x}(t)+B u\left(t-\hat{\delta}_{1}(t)+\epsilon\right)-L\left(y\left(t-\delta_{2}(t)\right)-\hat{y}\left(t-\delta_{2}(t)-\epsilon\right)\right), \\
& \hat{y}(t)=C \hat{x}(t),
\end{aligned}
$$

where the features of the system lead to $h_{m} \leq \delta_{i}(t) \leq h_{M}+T$ for $i=1,2$. Equivalently, if the average delay $\delta\left(h_{m}, h_{M}, T\right)=\left(h_{M}+T+h_{m}\right) / 2$ and the maximum delay amplitude $\mu\left(h_{m}, h_{M}, T\right)=\left(h_{M}+T-h_{m}\right) / 2$ is used, then:

$$
\delta-\mu \leq \delta_{i}(t) \leq \delta+\mu, \quad \forall i=1,2 .
$$

According to (6) and (7) and for given $k$ and any $t \in\left[t_{1, k}^{r}+h_{m}, t_{1, k+1}^{r}+h_{m}[\right.$, there exist $\hat{k}$ and $k^{\prime}$ such that the global remote system is governed by:

$$
\begin{aligned}
& \dot{x}(t)=\left(A+\Delta_{\gamma} A\right) x(t)+\left(B+\Delta_{\gamma} B\right) K \hat{x}\left(t_{1, k}\right), \\
& \dot{\hat{x}}(t)=A \hat{x}(t)+B K \hat{x}\left(t_{1, \hat{k}}-\epsilon\right)-\Delta_{\gamma} L C x\left(t_{2, k^{\prime}}\right)-L C\left(x\left(t_{2, k^{\prime}}\right)-\hat{x}\left(t_{2, k^{\prime}}+\epsilon\right)\right) .
\end{aligned}
$$


Rewriting the equations with the error $e(t)=x(t)-\hat{x}(t)$, the dynamics become:

$$
\begin{aligned}
\dot{x}(t) & =\left(A+\Delta_{\gamma} A\right) x(t)+\left(B+\Delta_{\gamma} B\right) K\left(x\left(t_{1, k}\right)-e\left(t_{1, k}\right)\right) \\
\dot{e}(t) & =A e(t)+L C e\left(t_{2, k^{\prime}}\right)+\Delta A x(t)+\Delta B K\left(x\left(t_{1, k}\right)-e\left(t_{1, k}\right)\right)+\Delta_{\gamma} L C x\left(t_{2, k^{\prime}}\right) \\
& -B K \int_{t_{1, k}}^{t_{1, \hat{k}}+\epsilon}[\dot{x}(s)-\dot{e}(s)] d s+L C \int_{t_{2, k^{\prime}}-\epsilon}^{t_{2, k^{\prime}}}[\dot{x}(s)-\dot{e}(s)] d s .
\end{aligned}
$$

Applying the input delay representation [6] yields:

$$
\begin{aligned}
\dot{x}(t) & =\left(A+\Delta_{\gamma} A\right) x(t)+\left(B+\Delta_{\gamma} B\right) K x\left(t-\delta_{1}\right)-\Delta_{\gamma} B K e\left(t-\delta_{1}\right) \\
\dot{e}(t) & =A e(t)+\Delta_{\gamma} A x(t)+\Delta_{\gamma} B K\left(x\left(t-\delta_{1}\right)-e\left(t-\delta_{1}\right)\right)+L \Delta_{\gamma} C x\left(t-\delta_{2}\right) \\
& +L C e\left(t-\delta_{2}\right)-B K \int_{t-\delta_{1}}^{t-\hat{\delta}_{1}+\epsilon}[\dot{x}(s)-\dot{e}(s)] d s+L C \int_{t-\delta_{2}-\epsilon}^{t-\delta_{2}}[\dot{x}(s)-\dot{e}(s)] d s
\end{aligned}
$$

with $\delta_{1}(t)=t-t_{1, k}$ and $\delta_{2}(t)=t-t_{2, k^{\prime}}$. From the fact that the communication delays belong to the interval $\left[h_{m}, h_{M}\right]$ where $h_{m}$ and $h_{M}$ are given by the network properties. Then the condition (9) on the delays still holds.

In an ideal case, ie. $\epsilon=0$ (from A2, synchronized case), the C2P delays are assumed to be well known, ie. $\delta_{1}(t)=\hat{\delta}_{1}(t)$ (see [13]) and the model is assumed to be perfectly known and constant $(\gamma=0)$. For this ideal case, Theorem 2 and 3 from [13] deliver controller and observer gains, since the global system is rewritten using the error vector $e(t)=x(t)-\hat{x}(t)$ as:

$$
\begin{aligned}
& \dot{x}(t)=A x(t)+B K x\left(t-\delta_{1}(t)\right)-B K e\left(t-\delta_{1}(t)\right) \\
& \dot{e}(t)=A e(t)+L C e\left(t-\delta_{2}(t)\right)
\end{aligned}
$$

\subsection{Stability Criteria}

It is now accepted that $\delta_{1}(t) \neq \hat{\delta}_{1}(t)$ and $\epsilon \neq 0$. The stability of the controller and of the observer is not ensured anymore by Theorem 2 and 3 in [13], as $\epsilon \neq 0$ leads error in the delay measurement. As in equation (11), there are interconnection terms between the two variables $x$ and $e$, a separation principle is no longer applicable to prove the global stabilization. The stability proof requires to consider now both variables simultaneously.

Theorem 1. For given $K$ and $L$, suppose that, there exists for $q$ representing the subscript $x$ or $e$, positive definite matrices : $P_{q 1}, S_{q}, R_{q a}, R_{q \epsilon}, S_{x e}, Q_{x e}$ and $R_{b}$ and matrices of size $n \times n: P_{q 2}, P_{q 3}, Z_{q l}$ for $l=1,2,3, Y_{q l^{\prime}}$ for $l^{\prime}=1,2$ such that the following LMI's hold:

$$
\begin{gathered}
{\left[\begin{array}{ccccc}
\Theta_{x}^{i} & \Theta_{x 12}^{i} & \mu P_{x}^{T} A_{K}^{i} & P_{x}^{T} A_{K}^{i} & \mu P_{x}^{T} A_{K}^{i} \\
* & -S_{x}+2 R_{b} & 0 & 0 & 0 \\
* & * & -\mu R_{x a} & 0 & 0 \\
* & * & * & -S_{x e} & 0 \\
* & * & * & * & -\mu R_{b}
\end{array}\right]<0,} \\
{\left[\begin{array}{cccc}
\Pi^{i} & P_{e}^{T}\left[\begin{array}{c}
0 \\
\gamma A_{i}
\end{array}\right] & \alpha P_{e}^{T}\left[\begin{array}{c}
0 \\
\gamma B_{i} K
\end{array}\right] & (1+\mu) P_{e}^{T}\left[\begin{array}{c}
0 \\
\gamma L C_{i}
\end{array}\right] \\
* & 0 & 0 & 0 \\
* & -Q_{x e} & 0 & 0 \\
* & * & -\alpha R_{b} & 0 \\
* & * & -(1+\mu) R_{b}
\end{array}\right]<0,}
\end{gathered}
$$




$$
\left[\begin{array}{ccc}
R_{q} & Y_{q 1} & Y_{q 2} \\
* & Z_{q 1} & Z_{q 2} \\
* & * & Z_{q 3}
\end{array}\right] \geq 0, \quad q \in\{x, e\}
$$

where $\alpha=(1+2 \mu), \beta=2(\mu+\bar{\epsilon}), P_{q}=\left[\begin{array}{cc}P_{q 1} & 0 \\ P_{q 2} & P_{q 3}\end{array}\right]$ and

$$
\begin{aligned}
& \Pi^{i}=\left[\begin{array}{cccccccc}
\Theta_{e} & \Theta_{e 12}^{i} & \mu P_{e}^{T} A_{L} & \bar{\epsilon} P_{e}^{T} A_{L} & \bar{\epsilon} P_{e}^{T} A_{L} & \beta P_{e}^{T} A_{K} & \beta P_{e}^{T} A_{K} \\
* & -S_{e}+S_{x e} & 0 & 0 & 0 & 0 & 0 \\
* & * & -\mu R_{e a} & 0 & 0 & 0 & 0 \\
* & * & * & -\bar{\epsilon} R_{e \epsilon} & 0 & 0 & 0 \\
* & * & * & * & -\bar{\epsilon} R_{x \epsilon} & 0 & 0 \\
* & * & * & * & * & -\beta R_{e \epsilon} & 0 \\
* & * & * & * & * & * & -\beta R_{x \epsilon}
\end{array}\right] \\
& \Theta_{x 12}=P_{x}^{T} A_{K}^{i}-\left[\begin{array}{c}
Y_{x 1}^{T} \\
Y_{x 2}^{T}
\end{array}\right], \quad \Theta_{e 12}^{*}=P_{e}^{T}\left[\begin{array}{c}
-\beta R_{x \epsilon} \\
L C-\gamma B_{i} K
\end{array}\right]-\left[\begin{array}{c}
Y_{e 1}^{T} \\
Y_{e 2}^{T}
\end{array}\right], \\
& \Theta_{x}^{i}=\Theta_{x}^{n i}+\left[\begin{array}{cc}
Q_{x e} & 0 \\
0 & 2 \beta R_{x \epsilon}+4 \mu R_{b}
\end{array}\right], \quad \Theta_{e}=\Theta_{e}^{n}+\left[\begin{array}{cc}
0 & 0 \\
0 & 2 \beta R_{e \epsilon}+4 \mu R_{b}
\end{array}\right], \\
& \Theta_{x}^{n i}=P_{x}^{T}\left[\begin{array}{cc}
0 & I \\
\bar{A}_{i} & -I
\end{array}\right]+\left[\begin{array}{cc}
0 & I \\
\bar{A}_{i} & -I
\end{array}\right]^{T} P_{x}+\left[\begin{array}{cc}
S_{x}+Y_{x 1}+Y_{x 1}^{T}+\delta Z_{x 1} & Y_{x 2}+\delta Z_{x 2} \\
& \delta R_{x}+2 \mu R_{x a}+\delta Z_{x 3}
\end{array}\right], \\
& \Theta_{e}^{n}=P_{e}^{T}\left[\begin{array}{cc}
0 & I \\
A & -I
\end{array}\right]+\left[\begin{array}{cc}
0 & I \\
A & -I
\end{array}\right]^{T} P_{e}+\left[\begin{array}{cc}
S_{e}+Y_{e 1}+Y_{e 1}^{T}+\delta Z_{e 1} & Y_{e 2}+\delta Z_{e 2} \\
* & \delta R_{e}+2 \mu R_{e a}+\delta Z_{e 3}
\end{array}\right], \\
& \text { and where } A_{K}=\left[\begin{array}{c}
0 \\
B K
\end{array}\right], A_{K}^{i}=\left[\begin{array}{c}
0 \\
\bar{B}_{i} K
\end{array}\right] \text { and } A_{L}=\left[\begin{array}{c}
0 \\
L C
\end{array}\right] \text {. }
\end{aligned}
$$

Then, the NCS (10) is asymptotic stable.

The proof of Theorem 3 is given in the appendix.

Remark 1. Theorem 1 guarantees the robust stability of the global remote to be guaranteed system with respect to the synchronization error and for observer and controller gains given in [13]. Since the problems of designing observer and controller gains are dual, to develop constructive LMI's is not straightforward.

\section{Application to a mobile robot}

This study is illustrated on the model of a mobile robot (Slave) which can move in one direction. The identification phase gives the following dynamics:

$$
\left\{\begin{array}{l}
\dot{x}=\left[\begin{array}{cc}
0 & 1 \\
0 & -11,32-\zeta \gamma
\end{array}\right] x+\left[\begin{array}{c}
0 \\
-11,32+\zeta \gamma
\end{array}\right] u\left(t-\delta_{1}\right), \\
y=\left[\begin{array}{ll}
1+\zeta \gamma / 10 & 0
\end{array}\right] x
\end{array}\right.
$$

where the scalar function $\zeta(t)$ lies in $[-1,1]$ and is taken as $\zeta(t)=\sin (6 t)$. The characteristics of transmission delays in a classical network (between Lens and Lille in France $(50 \mathrm{~km}))$ allows $h_{m}=0,1 s$ and $h_{M}=0.4 s$. Consider now that the bandwidth of the network allows the sampling period as $T=0.1 \mathrm{~s}$ to be defined. For these values, Theorems 2 and 3 in [13] produce the following gains $L=[-0.9119-0.0726]^{T}$ and $K=[-0.9125-0.0801]$. This gains ensures that, in the ideal case the remote system is $\alpha$-stable for $\alpha_{x}=\alpha_{e}=1.05$. Theorem 1 ensures that, with these features, the global system is asymptotically stable and robust without any time-varying synchronization error less than $\bar{\epsilon}=0.04 \mathrm{~s}$ in (5) for $\gamma=0$. Figure 3 shows the the maximal admissive $\bar{\epsilon}$ for greater values 


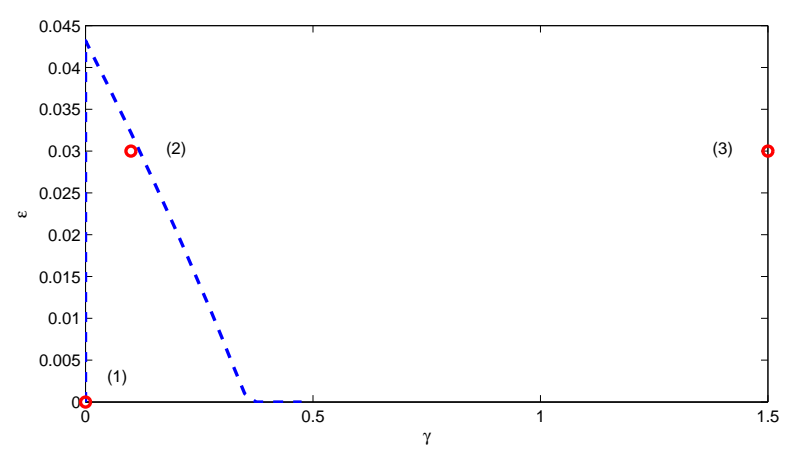

Fig. 3: Maximal synchronization error with respect to the disturbances

of $\gamma$. Moreover it guarantees asymptotic stability of the global system without the introduction of a buffer in the controller.

Figure 4a shows the simulation results for $\gamma=0.1$ and $\epsilon=0.03$ (point (2) in Figure 3). The state of the process and the sampled input and output are provided. It can be seen that the state convergence to the reference. The stability of the system despite the synchronization error and the parameters uncertainties is ensured.

Figure $4 \mathrm{~b}$ present simulations for $\gamma=0$ and $\epsilon=0$ (point (1)) and for $\gamma=1.5$ and $\epsilon=0.03$ (point (3)). In comparison to Figure 4a, the results for (1) are closed to the ones obtained for (2). Concerning (3), Theorem 1 does not ensure the stability. However the controller still stabilize the system. It means that the conditions from Theorem 1 are conservative. Further results would investigate in reducing the conservativeness of the stability conditions.

\section{Concluding remarks}

This paper presents a strategy for an observer-based control of a networked controlled systems under synchronization erros. No buffering technique was involved, which allows using the available information as soon as received. Various perturbations were dealt with jittery, non-symmetric and unpredictable delays, synchronization error, aperiodic sampling (real-time) and uncertainties in the model. A remaining assumption in [13] which is that the clocks have to be synchronized is not required anymore.

A characteristic feature of this control strategy is to consider that the observer based controller runs in continuous time (i.e., with small computation step) whereas the process provides discrete-time measurements. Thus, the observer keeps on providing a continuous estimation of the current state, even if the data are not sent continuously. 

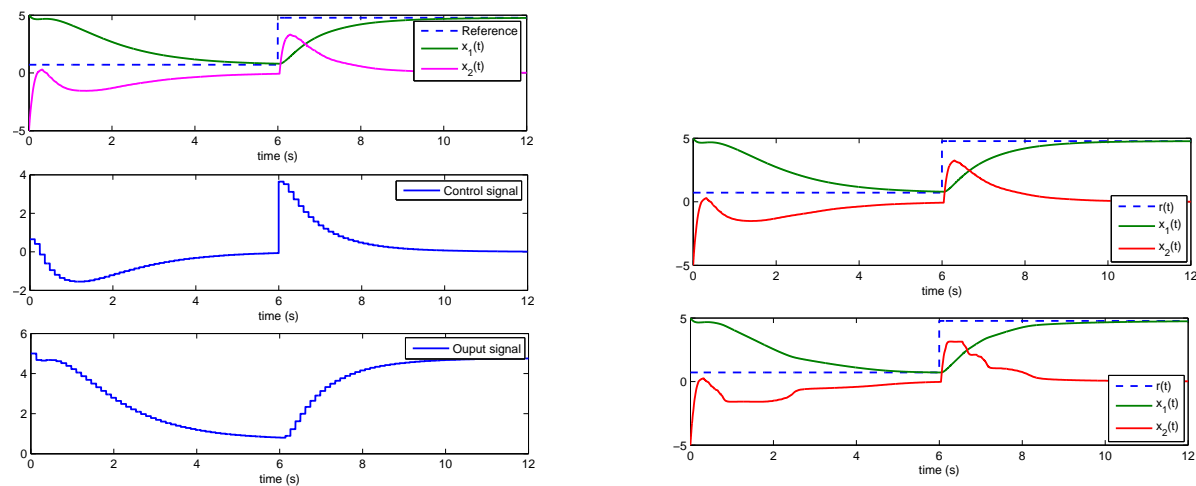

(a) Simulation results for $\gamma=0.1$ and $\epsilon=0.1$ (2)

(b) Simulations for (1) and (3)

Fig. 4: Simulation results

The proposed conditions are conservative. New and less conservative results which guarantee stability of system with sampled-data control recently appears and might help in reducing the conservativeness. It would be interesting to apply these new technics on the present system.

\section{References}

1. C.T. Abdallah, Delay effect in the networked control of mobile robot, in Application in Time-Delay Systems, Edt J. Chiasson and J.-J. Loiseau, LNCIS 352, SpringerVerlag, Berlin Heidelberg, 2007.

2. J.M. Azorin, O. Reinoso, J.M. Sabater, R.P. Neco and R. Aracil, Dynamic analysis for a teleoparation system with time delay, Proceeding of Conference on Control Applications, pp 1170-1175, June 2003.

3. A. Eusebi and C. Melchiorri, Force-Reflecting telemanipulators with Time-delay: Stability Analysis and control design, IEEE trans. on Robotics and Automation, vol. 14(4), pp 635-640, 1998.

4. W.R. Ferrel, Remote manipulation with transmission delay, IEEE Trans. on Human Factors in Electronics, vol. HFE-6, pp 24-32, 1965.

5. N.M. Freris and P.R. Kumar, Fundamental Limits on Synchronization of Affine Clocks in Networks, Proceedings of the 46th IEEE Conference on Decision and Control, New Orleans, LA, USA, Dec. 12-14, 2007.

6. E. Fridman, A. Seuret and J.-P. Richard, Robust Sampled-Data Stabilization of Linear Systems: An Input Delay Approach, Automatica, vol. 40(8), pp 1141-1146, 2004.

7. E. Fridman and U. Shaked, A descriptor system approach to $H^{\infty}$ control of linear time-delay systems, IEEE Trans. on Automatic Control, vol. 47(2), pp 253-270, 2002. 
8. J.P. Hespanha, P. Naghshtabrizi and Y. Xu, A survey of recent results in networked control systems, Proceedings of the IEEE Vol. 95(1), pp 138-162, January 2007.

9. L.A. Motestruque and P.J. Antsaklis, Stability of model-based networked control system with time-varying transmission time, IEEE Tans. on Automatic Control, vol. 49(9), pp 1562-1572, 2004.

10. S.-I. Niculescu, Delay Effects on Stability. A Robust Control Approach, SpringerVerlag, 2001.

11. J.-P. Richard, Time delay systems: an overview of some recent advances and open problems, Automatica, vol. 39(10), pp 1667-1694, 2003.

12. A. Seuret, E. Fridman and J.-P. Richard, Sampled-data exponential stabilization of neutral systems with input and state delays, IEEE MED, $13^{\text {th }}$ Mediterranean Conference on Control and Automation, Cyprus, June 2005.

13. A. Seuret, F. Michaut, J.-P. Richard and T. Divoux, Networked Control using GPS Synchronization, American Control Conference, Minneapolis, US, June, 2006.

14. E. Witrant, C. Canudas de Wit, D. Georges and M. Alamir, Remote output stabilization via communication networks with a distributed control law, IEEE Trans. on Automatic Control, vol. 52(8), 2007, pp 1480-1485.

15. D.Yue, Q.-L. Han and C.Peng, State feedback controller design for networked control systems, IEEE Trans. on Automatic Control, vol. 51(11), pp 640-644, 2004.

16. S. Zampieri, "A survey of recent results in Networked Control Systems," in Proc. of the $17^{\text {th }}$ IFAC World Congress, Seoul, Korea, July 2008, pp. 2886-2894.

\section{A Proof of Theorem 1}

To analyze the asymptotic stability property of such a system, equations (11) are rewritten by using the descriptor representation [7] with $\bar{x}(t)=\operatorname{col}\{x(t), \dot{x}(t)\}$, $\bar{e}(t)=\operatorname{col}\{e(t), \dot{e}(t)\}$. In this section, when there is no confusion, any function considered at time ' $t$ ' will be written without ' $(t)$ '. Consider the LyapunovKrasovskii (LK) functional:

$$
V=V_{x n}+V_{x a}+V_{x \epsilon}+V_{e n}+V_{e a}+V_{e \epsilon}+V_{x e}
$$

where the sub-LK functionals are, for q representing the subscript of the variables ' $\mathrm{x}$ ' and 'e':

$$
\begin{aligned}
V_{q n}= & \bar{q}^{T} E P_{q} \bar{q}+\int_{-\delta}^{0} \int_{t+\theta}^{t} \dot{q}^{T}(s) R_{q} \dot{q}(s) d s d \theta \\
& +\int_{t-\delta}^{t} q^{T}(s) S_{q} q(s) d s, \\
V_{q a}= & \int_{-\mu}^{\mu} \int_{t+\theta-\delta}^{t} \dot{q}^{T}(s) R_{q a} \dot{q}(s) d s d \theta, \\
V_{q \epsilon}= & 2 \int_{-\mu \bar{\epsilon}}^{\mu+\bar{\epsilon}} \int_{t+\theta-\delta}^{t} \dot{q}^{T}(s) R_{q \epsilon} \dot{q}(s) d s d \theta \\
V_{q b}= & 2 \int_{-\mu}^{\mu} \int_{t+\theta-\delta}^{t} \dot{q}^{T}(s) R_{b} \dot{q}(s) d s d \theta
\end{aligned}
$$

with $E=\operatorname{diag}\left\{I_{n}, 0\right\}$ and $P_{x}, P_{e}$ defined in Theorem 1 .

The signification of each sub-LK functional has to be explain. The first functionals $V_{x n}$ and $V_{e n}$ deal with the stability of the Slave and the observer systems subject to the constant delay $\delta$ while $V_{x a}$ and $V_{e a}$ refer to the disturbances due to the delay variations. Even if the functionals do not explicitly depend on each time varying delay, it will be considered both different delays $\delta_{1}$ and $\delta_{2}$. The functionals $V_{q \epsilon}$ are concerned with synchronization errors. The last functionals 
$V_{q b}$ deals with the interconnection between the variables $x$ and $e$. Consider as a first step, the polytopic representation of the dynamics in $x$ :

$$
\dot{x}=\sum_{i=1}^{N} \lambda_{i}\left\{\bar{A}_{i} x+\bar{B}_{i} K\left(x\left(t-\delta_{1}\right)-e\left(t-\delta_{1}\right)\right)\right\}
$$

where $\bar{A}_{i}=A+\gamma A_{i}$ and $\bar{B}_{i}=B+\gamma B_{i}$. According to Theorem 2 in [12], if LMI (14) holds for ' $q=x^{\prime}$ and for all vertices of the polytopic system, the following inequality holds:

$$
\dot{V}_{x n}+\dot{V}_{x a} \leq \sum_{i=1}^{N} \lambda_{i}\left\{\xi_{x}^{T}\left[\begin{array}{cc}
\Psi_{x 1}^{i} & \Theta_{x 12}^{i} \\
* & -S_{x}
\end{array}\right] \xi_{x}+\eta_{x}^{i}\right\}
$$

where $\xi_{x}=\operatorname{col}\{x, \dot{x}, x(t-\delta)\}$ and:

$$
\eta_{x}^{i}=-2 \bar{x}^{T} P_{x}^{T} A_{K}^{i} e\left(t-\delta_{1}\right), \quad \Psi_{x 1}^{i}=\Theta_{x}^{n i}+\mu P_{x}^{T} A_{K}^{i} R_{x a}^{-1} A_{K}^{i T} P_{x} .
$$

Using the Leibnitz formula and a classical LMI bounding, it yields, for $i=1,2$ :

$$
\begin{aligned}
& \eta_{x}^{i} \leq \bar{x}^{T} P_{x}^{T} A_{K}^{i}\left(S_{x e}^{-1}+\mu R_{b}^{-1}\right) A_{K}^{i T} P_{x} \bar{x} \\
& \quad+e^{T}(t-\delta) S_{x e} e(t-\delta)+\left|\int_{t-\delta_{1}}^{t-\delta} \dot{e}^{T}(s) R_{b} \dot{e}(s) d s\right|
\end{aligned}
$$

where $S_{x e}$ and $R_{b}$ are positive definite matrices which represent the presence of the error vector in the state equation. Then, the following inequality holds:

$$
\begin{aligned}
& \dot{V}_{x n}+\dot{V}_{x a} \leq \sum_{i=1}^{N} \lambda_{i}\left\{\xi_{x}^{T}\left[\begin{array}{cc}
\Psi_{x 2}^{n i} & \Theta_{x 12}^{i} \\
* & -S_{x}
\end{array}\right] \xi_{x}\right\} \\
& +e^{T}(t-\delta) S_{x e} e(t-\delta)+\left|\int_{t-\delta_{1}}^{t-\delta} \dot{e}^{T}(s) R_{b} \dot{e}(s) d s\right|,
\end{aligned}
$$

where $\Psi_{x 2}^{n i}=\Theta_{x}^{n i}+P_{x}^{T} A_{K}^{i}\left(S_{x e}^{-1}+\mu R_{x a}^{-1}+\mu R_{b}^{-1}\right) A_{K}^{i T} P_{x}$. Concerning the errors dynamics, differentiating $V_{e n}+V_{e a}$ along the trajectory of (11) and assuming that LMI (14) holds with $q=e$ yields:

$$
\begin{aligned}
\dot{V}_{e n}+ & \dot{V}_{e a} \leq \sum_{i=1}^{N} \lambda_{i}\left\{\xi_{e}^{T}\left[\begin{array}{cc}
\Psi_{e 1} & P_{e}^{T} A_{L}-Y_{e}^{T} \\
* & -S_{e}
\end{array}\right] \xi_{e}-\eta_{e 1}^{x}\right. \\
& \left.+\eta_{e 1}^{e}-\eta_{e 2}^{x}+\eta_{e 2}^{e}+\eta_{\Delta A}^{x i}+\eta_{\Delta B}^{x i}+\eta_{\Delta B}^{e i}+\eta_{\Delta C}^{x i}\right\}
\end{aligned}
$$

where $\xi_{e}=\operatorname{col}\{e, \dot{e}, e(t-\delta)\}$ and where

$$
\begin{aligned}
& \Psi_{e 1}=\Theta_{e}^{n}+\mu P_{e}^{T} A_{L} R_{e a}^{-1} A_{L}^{i T} P_{e}, \quad \eta_{e 1}^{q}=2 \bar{e}^{T} P_{e}^{T} A_{K} \int_{t_{1, k}}^{t_{1, \hat{k}}+\epsilon} \dot{q}(s) d s,
\end{aligned}
$$

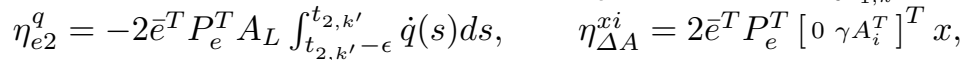

$$
\begin{aligned}
& \eta_{\Delta B}^{x i}=2 \bar{e}^{T} P_{e}^{T}\left[0 \gamma\left(B_{i} K\right)^{T}\right]^{T} x\left(t-\delta_{1}\right), \eta_{\Delta B}^{e i}=-2 \bar{e}^{T} P_{e}^{T}\left[0 \gamma\left(B_{i} K\right) T\right]^{T} e\left(t-\delta_{1}\right), \\
& \eta_{\Delta C}^{x i}=2 \bar{e}^{T} P_{e}^{T}\left[0 \gamma\left(L C_{i}\right)^{T}\right]^{T} x\left(t-\delta_{1}\right),
\end{aligned}
$$

with $q$ representing either $x$ or $e$. Note that the functions $\eta_{e i}^{q}$, for $q={ }^{\text {' }} x$ ', ' $e$ ' and $i=1,2$ correspond to the disturbance due to the synchronization error. Consider $i=1$ : Noting that from assumption $\mathbf{A} 4$, inequality $t_{1, \hat{k}}+\epsilon-t_{1, k} \leq \bar{\epsilon}+2 \mu$ holds, then a classical bounding leads to:

$$
\eta_{q 1}^{x} \leq(\bar{\epsilon}+2 \mu) \bar{e}^{T} P_{e}^{T} A_{K} R_{q \epsilon}^{-1} A_{K}^{T} P_{e} \bar{e}+\int_{t_{1, k}}^{t_{1, \hat{k}}+\epsilon} \dot{q}^{T}(s) R_{q \epsilon} \dot{q}(s) d s .
$$


By the same way, the following inequalities hold:

$$
\eta_{e 2}^{q} \leq \bar{\epsilon} \bar{e}^{T} P_{e}^{T} A_{L} R_{q \epsilon}^{-1} A_{L}^{T} P_{e} \bar{e}+\int_{t_{2, k^{\prime}}-\epsilon}^{t_{2, k^{\prime}}} \dot{q}^{T}(s) R_{q \epsilon} \dot{q}(s) d s .
$$

Following the same method as in (19), the following inequalities hold:

$$
\begin{aligned}
\eta_{\Delta A}^{x i} & \leq \bar{e}^{T} P_{e}^{T}\left[\begin{array}{c}
0 \\
\gamma A_{i}
\end{array}\right] Q_{x e}^{-1}\left[\begin{array}{c}
0 \\
\gamma A_{i}
\end{array}\right]^{T} P_{e} \bar{e}+x^{T} Q_{x e} x, \\
\eta_{\Delta B}^{x i} & \leq(1+\mu) \bar{e}^{T} P_{e}^{T}\left[\begin{array}{c}
0 \\
\gamma B_{i} K
\end{array}\right] R_{b}^{-1}\left[\begin{array}{c}
0 \\
\gamma B_{i} K
\end{array}\right]^{T} P_{e} \bar{e} \\
& +x^{T}(t-\delta) R_{b} x(t-\delta)+\left|\int_{t-\delta_{1}}^{t-\delta} \dot{x}^{T}(s) R_{b} \dot{x}(s) d s\right|, \\
\eta_{\Delta B}^{e i} & \leq \mu \bar{e}^{T} P_{e}^{T}\left[\begin{array}{c}
0 \\
\gamma B_{i} K
\end{array}\right] R_{b}^{-1}\left[\begin{array}{c}
0 \\
\gamma B_{i} K
\end{array}\right]^{T} P_{e} \bar{e} \\
& -2 \bar{e}^{T} P_{e}^{T}\left[\begin{array}{c}
0 \\
\gamma B_{i} K
\end{array}\right] e(t-\delta)+\left|\int_{t-\delta_{1}}^{t-\delta} \dot{e}^{T}(s) R_{b} \dot{e}(s) d s\right|, \\
\eta_{\Delta C}^{x i} & \leq(1+\mu) \bar{e}^{T} P_{e}^{T}\left[\begin{array}{c}
0 \\
\gamma L C_{i}
\end{array}\right] R_{b}^{-1}\left[\begin{array}{c}
0 \\
\gamma L C_{i}
\end{array}\right]^{T} P_{e} \bar{e} \\
& +x^{T}(t-\delta) R_{b} x(t-\delta)+\left|\int_{t-\delta_{2}}^{t-\delta} \dot{x}^{T}(s) R_{b} \dot{x}(s) d s\right| .
\end{aligned}
$$

Finally, the following inequality holds:

$$
\begin{aligned}
& \dot{V}_{e n}+\dot{V}_{e a} \leq \xi_{e}^{T}\left[\begin{array}{cc}
\Psi_{e 2}^{n} & \Theta_{12}^{e i} \\
* & -S_{e}+R_{b}
\end{array}\right] \xi_{e}+x^{T} Q_{x e} x \\
& \quad+2 x^{T}(t-\delta) R_{b} x(t-\delta)-2 \bar{e}^{T} P_{e}^{T}\left[\begin{array}{c}
0 \\
\gamma B_{i} K
\end{array}\right] e(t-\delta) \\
& \quad+\left|\int_{t-\delta_{2}}^{t-\delta} \dot{x}^{T}(s) R_{b} \dot{x}(s) d s\right|+\sum_{q=x, e}\left\{\left|\int_{t-\delta_{1}}^{t-\delta} \dot{q}^{T}(s) R_{b} \dot{q}(s) d s\right|\right. \\
& \left.\left.\quad+\int_{t_{1, k}}^{t_{1, \hat{k}}+\epsilon} \dot{q}^{T}(s) R_{q p} \dot{q}(s) d s+\int_{t_{2, k^{\prime}-\epsilon}}^{t_{2, k^{\prime}}} \dot{q}^{T}(s) R_{q p} \dot{q}(s) d s\right)\right\},
\end{aligned}
$$

where

$$
\begin{aligned}
& \Psi_{e 2}^{n}=\Theta_{e}^{n}+P_{e}^{T} A_{L}\left(\mu R_{e a}+\bar{\epsilon} R_{x \epsilon}^{-1}+\bar{\epsilon} R_{e \epsilon}^{-1}\right)^{-1} A_{L}^{T} P_{e} \\
& \quad+\beta P_{e}^{T} A_{K}\left(R_{x \epsilon}^{-1}+R_{e \epsilon}^{-1}\right) A_{K}^{T} P_{e}+P_{e}^{T}\left[\begin{array}{c}
0 \\
\gamma A_{i}
\end{array}\right] Q_{x e}^{-1}\left[\begin{array}{c}
0 \\
\gamma A_{i}
\end{array}\right]^{T} P_{e} \\
& \quad+\alpha P_{e}^{T}\left[\begin{array}{c}
0 \\
\gamma B_{i} K
\end{array}\right] R_{b}^{-1}\left[\begin{array}{c}
0 \\
\gamma B_{i} K
\end{array}\right]^{T} P_{e}+(1+\mu) P_{e}^{T}\left[\begin{array}{c}
0 \\
\gamma L C_{i}
\end{array}\right] R_{b}^{-1}\left[\begin{array}{c}
0 \\
\gamma L C_{i}
\end{array}\right]^{T} P_{e} .
\end{aligned}
$$

Differentiating $V_{x \epsilon}, V_{e \epsilon}, V_{x b}$ and $V_{e b}$ leads to:

$$
\begin{aligned}
& \dot{V}_{q \epsilon}=2 \beta \dot{q}^{T} R_{q \epsilon} \dot{q}-2 \int_{t-\delta-\mu-\bar{\epsilon}}^{t-\delta+\mu+\bar{q}} \dot{q}^{T}(s) R_{x \epsilon} \dot{q}(s) d s \\
& \dot{V}_{q b}=4 \mu \dot{q}^{T} R_{b} \dot{q}-2 \int_{t-\delta-\mu}^{t-\delta+\mu} \dot{q}^{T}(s) R_{b} \dot{q}(s) d s,
\end{aligned}
$$

Combining (20), (25) and (26) and noting that the sum of the negative integrals in (26) with the integrals from (23) is negative, the following inequality holds:

$$
\dot{V} \leq \sum_{i=1}^{N} \lambda_{i}\left\{\xi_{x}^{T}\left[\begin{array}{cc}
\Psi_{x}^{i} & \Theta_{12}^{x i} \\
* & -S_{x}+R e x
\end{array}\right] \xi_{x}+\xi_{e}^{T}\left[\begin{array}{cc}
\Psi_{e} & \Theta_{12}^{e i} \\
* & -S_{e}+S_{x e}
\end{array}\right] \xi_{e}\right\}
$$

where $\Psi_{x}^{i}=\Psi_{x 2}^{n i}+\left[\begin{array}{ll}0 & 0 \\ 0 & 2 \beta R_{x \epsilon}+4 \mu R_{b}\end{array}\right]$, and $\Psi_{e}=\Psi_{e}^{n}+\left[\begin{array}{ll}0 & 0 \\ 0 & 2 \beta R_{e \epsilon}+4 \mu R_{b}\end{array}\right]$.

Then the Schur complement leads to the LMI's given in (12) and (13). Then LMI's from Theorem 1 are satisfied, the system (11) is asymptotically stable. 